\title{
Silicon-Integrated Hybrid-Cavity 850-nm VCSELs by Adhesive Bonding: Impact of Bonding Interface Thickness on Laser Performance
}

\author{
Emanuel P. Haglund, Student Member, IEEE, Sulakshna Kumari, Erik Haglund, Member, IEEE, Johan S. Gustavsson, \\ Roel G. Baets, Fellow, IEEE, Gunther Roelkens, Member, IEEE, and Anders Larsson, Fellow, IEEE
}

\begin{abstract}
The impact of bonding interface thickness on the performance of $850-\mathrm{nm}$ silicon-integrated hybrid-cavity verticalcavity surface-emitting lasers (HC-VCSELs) is investigated. The HC-VCSEL is constructed by attaching a III-V "half-VCSEL" to a dielectric distributed Bragg reflector on a Si substrate using ultrathin divinylsiloxane-bis-benzocyclobutene (DVS-BCB) adhesive bonding. The thickness of the bonding interface, defined by the DVS-BCB layer together with a thin $\mathrm{SiO}_{2}$ layer on the "halfVCSEL," can be used to tailor the performance, for e.g., maximum output power or modulation speed at a certain temperature, or temperature-stable performance. Here, we demonstrate an optical output power of 2.3 and $0.9 \mathrm{~mW}$, a modulation bandwidth of 10.0 and $6.4 \mathrm{GHz}$, and error-free data transmission up to 25 and $10 \mathrm{~Gb} / \mathrm{s}$ at an ambient temperature of 25 and $85^{\circ} \mathrm{C}$, respectively. The thermal impedance is found to be unaffected by the bonding interface thickness.
\end{abstract}

Index Terms-Heterogeneous integration, high-speed modulation, large signal modulation, optical interconnects, semiconductor lasers, silicon photonics, vertical-cavity surface-emitting laser (VCSEL).

\section{INTRODUCTION}

$\mathbf{T}$ HE integration of an efficient light source is considered the Holy Grail of silicon photonics (SiP) [1]. For shortwavelength $\mathrm{SiP}$ (i.e. silicon nitride $(\mathrm{SiN})$ waveguide circuits), integration concepts utilizing GaAs-based vertical-cavity surface-emitting laser (VCSEL) technology are attractive, since the small modal and gain volumes facilitate energy efficient operation with demonstrated power conversion efficiencies exceeding 60\% [2] for regular VCSELs. Such integration concepts can potentially enable fully integrated short-wavelength

Manuscript received September 27, 2016; revised November 21, 2016; accepted November 22, 2016. This work was supported in part by the Swedish Foundation for Strategic Research, in part by the FP7-ERC-InSpectra Advanced Grant, and in part by the European Union's Horizon 2020 research and innovation program under Grant 688519 (PIX4life).

E. P. Haglund, E. Haglund, J. S. Gustavsson, and A. Larsson are with the Photonics Laboratory, Department of Microtechnology and Nanoscience, Chalmers University of Technology, Göteborg SE-412 96, Sweden (e-mail: emanuel.haglund@chalmers.se; erik.haglund@chalmers.se; johan.gustavsson@chalmers.se; anders.larsson@chalmers.se).

S. Kumari, R. G. Baets, and G. Roelkens are with the Photonics Research Group, Center for Nano-and Biophotonics, Gent B-9000, Belgium (e-mail: sulakshna.kumari@ugent.be; roel.baets@ugent.be; gunther.roelkens@ ugent.be).

Color versions of one or more of the figures in this paper are available online at http://ieeexplore.ieee.org.

Digital Object Identifier 10.1109/JSTQE.2016.2633823

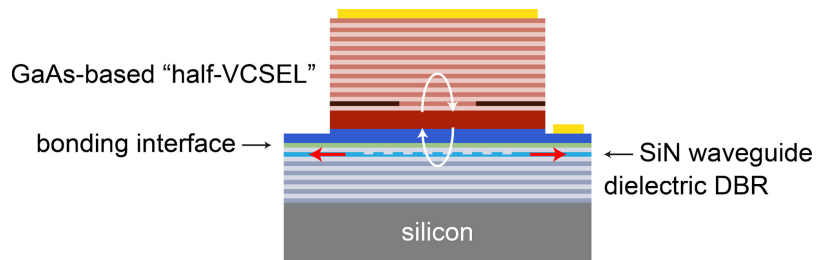

(a)

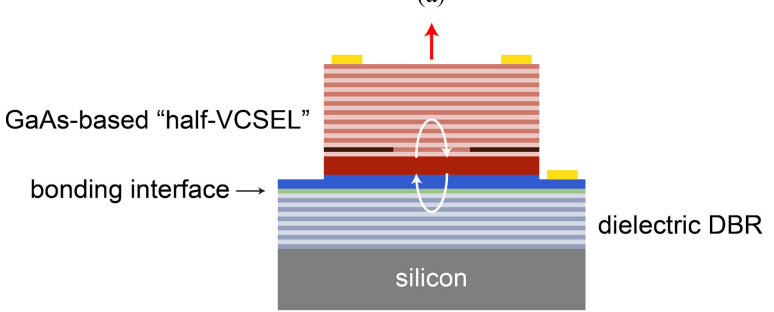

(b)

Fig. 1. (a) Hybrid vertical-cavity laser with in-plane emission, and (b) hybridcavity VCSEL.

photonic integrated circuits (PICs) for optical interconnects and bio-sensing applications.

To equip SiP ICs with integrated light sources, several techniques have been explored, including flip-chip bonding [3] and direct growth of III-V material on Si [4]. Flip-chip bonding requires stringent alignment whereas direct growth struggles to achieve high-quality material. An alternative technique is heterogeneous integration where a III-V epitaxial structure grown on its native substrate is bonded to the SiP IC using direct or adhesive bonding [5]. This technique has been used to demonstrate silicon-integrated InP-based hybrid vertical-cavity lasers for surface and in-plane emission [6]-[8]. The hybrid cavity, which implies that the standing wave optical field extends over both the III-V and the silicon-based parts of the cavity, provides an opportunity to tap off light to an in-plane waveguide using e.g. an intra-cavity waveguide with a weak diffraction [9], see Fig. 1(a).

Recently, we demonstrated silicon-integrated GaAs-based hybrid-cavity VCSELs (HC-VCSELs) with $1.6 \mathrm{~mW}$ output power at $845 \mathrm{~nm}$ [10] and capable of $20 \mathrm{~Gb} / \mathrm{s}$ error-free data transmission under direct current modulation [11]. Ultrathin divinylsiloxane-bis-benzocyclobutene (DVS-BCB) adhesive bonding was used to attach a III-V "half-VCSEL" to a dielectric distributed Bragg reflector (DBR) on Si, see Fig. 1(b). 


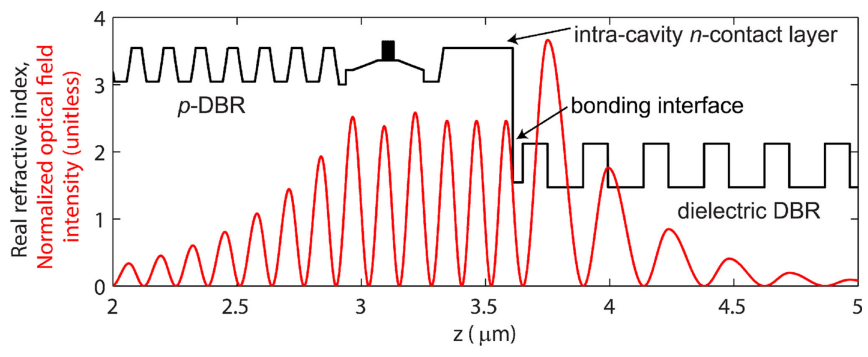

Fig. 2. Simulated standing-wave optical field intensity along the optical axis of the HC-VCSEL (red), together with the real refractive index profile (black).

While this device does not yet contain the intra-cavity waveguide with a diffraction grating for in-plane emission, and thus is surface emitting, it still allows the investigation and development of the integration concept and design.

In our previous work [10], [11], the output power and high temperature performance were limited by a too small detuning between the gain peak and the cavity resonance wavelengths. However, the hybrid-cavity concept offers the opportunity to control the detuning, and therefore the laser performance, by the thickness of the bonding interface, while in an ordinary VCSEL the detuning is set during epitaxial growth and cannot be adjusted in a post-growth process. Here, we present the results from an investigation where we have systematically varied the thickness of the bonding interface and monitored the impact on important laser performance parameters. We show, that the thickness can be chosen to optimize a certain performance parameter at a given temperature or to minimize the variation of performance with temperature. We also find that the thermal impedance of the HC-VCSEL is largely unaffected by the thickness of the bonding interface. Finally, we demonstrate errorfree data transmission at bit rates up to $25 \mathrm{~Gb} / \mathrm{s}$ and $10 \mathrm{~Gb} / \mathrm{s}$ at 25 and $85^{\circ} \mathrm{C}$, respectively.

The paper is organized as follows. Section II describes the concept and design of the HC-VCSEL and presents results from simulations. In Section III the device fabrication is outlined, while Section IV presents results from static and dynamic measurements. The paper is concluded in Section V.

\section{Design And Simulations}

The HC-VCSEL design is illustrated in Fig. 1(b). A plot showing the variations of the real part of the refractive index and the intensity of the optical standing wave along the optical axis of the cavity is presented in Fig. 2. To the left of the bonding interface is the III-V "half-VCSEL" consisting of an $n-\mathrm{Al}_{0.12} \mathrm{Ga}_{0.88} \mathrm{As}$ intra-cavity contact layer, an active region with five $4 \mathrm{~nm} \operatorname{In}_{0.10} \mathrm{Ga}_{0.90}$ As quantum wells (QWs) with photoluminescence (PL) peak measured at $840 \mathrm{~nm}$, a $p-\mathrm{Al}_{0.98} \mathrm{Ga}_{0.02} \mathrm{As}$ layer for the formation of an oxide aperture for transverse current and optical confinement, and a 23-pair $p-\mathrm{Al}_{0.90} \mathrm{Ga}_{0.10} \mathrm{As} / \mathrm{Al}_{0.12} \mathrm{Ga}_{0.88}$ As DBR. To the right of the interface is the 20-pair $\mathrm{SiO}_{2} / \mathrm{Ta}_{2} \mathrm{O}_{5}$ dielectric DBR on the $\mathrm{Si}$ substrate. The calculated reflectance (assuming lossless materials) is $>99.99 \%$ at the center wavelength of $845 \mathrm{~nm}$.
TABLE I

CAVITy PaRAmeters at a Resonance WaVELENGTH of $845 \mathrm{~nm}$

\begin{tabular}{lc}
\hline \hline Optical confinement factor & 0.0173 \\
Cold cavity Q & 16200 \\
Total loss $\left(\mathrm{ps}^{-1}\right)$ & 0.138 \\
Top DBR transmission loss $\left(\mathrm{ps}^{-1}\right)$ & 0.053 \\
Bottom DBR transmission loss $\left(\mathrm{ps}^{-1}\right)$ & $3 \cdot 10^{-5}$ \\
Intra-cavity loss $\left(\mathrm{ps}^{-1}\right)$ & 0.085 \\
Threshold QW gain $\left(\mathrm{cm}^{-1}\right)$ & 609 \\
Photon lifetime (ps) & 7.25 \\
\hline
\end{tabular}

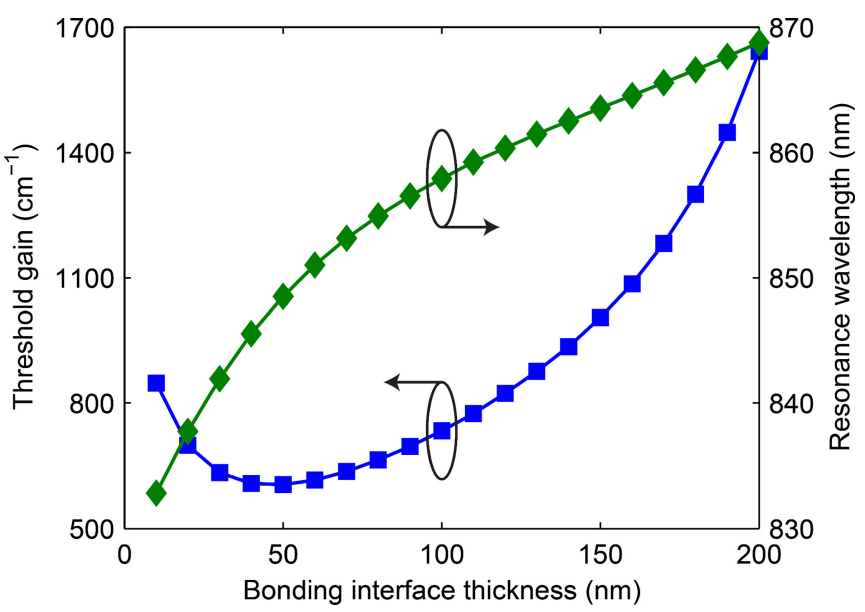

Fig. 3. Simulated dependence of resonance wavelength and threshold gain on bonding interface thickness.

The bonding interface is defined by a $\sim 40 \mathrm{~nm}$ DVS-BCB bonding layer and a thin $\mathrm{SiO}_{2}$ layer deposited on the dielectric DBR prior to bonding. The thickness of the $\mathrm{SiO}_{2}$ layer is used to control the interface thickness and therefore the cavity length, which sets the cavity resonance wavelength and the detuning between the gain peak (estimated at $852 \mathrm{~nm}$ from the PL wavelength) and resonance wavelengths. Detuning is an important design parameter as it controls the optical gain properties as well as the temperature dependence of important laser parameters since the gain peak red-shifts faster with temperature than the resonance wavelength [12]. The detuning can therefore be chosen for either optimized performance at a specific temperature or stable performance over temperature [13]. It should be noted however, that in a final design the desired detuning can be obtained with a bonding interface comprising only the DVS-BCB layer with appropriate thickness. The length of the cavity is $3 \lambda$ to accommodate an $n$-contact layer with sufficient thickness for low electrical series resistance.

The optical cavity properties were analyzed using a 1D effective index model [14]. Table I lists some important cavity parameters when the interface thickness is adjusted for a resonance wavelength of $845 \mathrm{~nm}$. The intra-cavity loss is due to free-carrier absorption in the $n$-contact layer and the $p$-DBR. The variations of QW threshold gain and cavity resonance wavelength with the thickness of the bonding interface are shown in Fig. 3. Over a $\sim 35 \mathrm{~nm}$ wavelength span $(830-865 \mathrm{~nm})$, the threshold gain is below $1000 \mathrm{~cm}^{-1}$, which should allow for low threshold currents. 


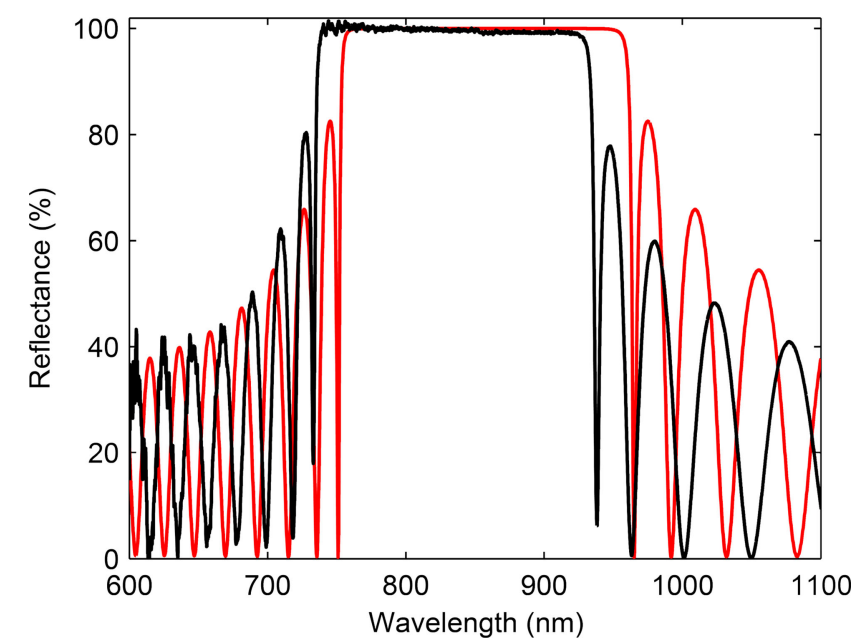

Fig. 4. Simulated (red) and measured (black) spectral reflectance for the 20-pair $\mathrm{SiO}_{2} / \mathrm{Ta}_{2} \mathrm{O}_{5}$ dielectric DBR.

\section{FABRICATION}

The 20-pair $\mathrm{SiO}_{2} / \mathrm{Ta}_{2} \mathrm{O}_{5}$ dielectric DBR was deposited on a polished Si substrate. A comparison of simulated and measured reflectance spectra (Fig. 4) shows good agreement in terms of the width of the stopband $(\sim 200 \mathrm{~nm})$ and a slight blue-shift of the measured center wavelength $(\sim 20 \mathrm{~nm})$ with respect to simulations.

The III-V "half-VCSEL" epitaxial structure was grown on a GaAs substrate by metal-organic chemical vapor deposition at IQE Europe Ltd. Dies $(8 \mathrm{~mm} \times 10 \mathrm{~mm})$ of the epitaxial structure were bonded to the dielectric DBR using ultra-thin DVS-BCB adhesive bonding [5]. The bonding process includes spin-coating of DVS-BCB:mesitylene on the dielectric DBR, which was partially cured before the "half-VCSEL" die was attached with the epitaxial structure facing the dielectric DBR. The die and dielectric DBR were clamped between two glass wafers and mounted in a wafer bonding tool where pressure was applied on the "half-VCSEL" die while the DVS-BCB bonding interface was fully cured. The bonding process results in a DVS-BCB thickness of $\sim 40 \mathrm{~nm}$, which is controllable within $\pm 10 \mathrm{~nm}$ and with a uniformity of $\pm 5 \mathrm{~nm}$ for both dies and wafers [5]. Prior to bonding, thin $\mathrm{SiO}_{2}$ layers with varying thickness were deposited on dielectric DBR samples by plasma-enhanced chemical vapor deposition (PECVD). This layer has similar refractive index as DVS-BCB and the thickness can be controlled with an accuracy of $5 \%$. The effects of thickness variation on performance is shown in Section IV. Four samples with an interface thickness of $35,65,125$, and $180 \mathrm{~nm}$ and cavity resonance wavelengths of $843,853,861$, and $867 \mathrm{~nm}$ were prepared, corresponding to a gain-to-resonance detuning of approximately +9 , $-1,-9$, and $-15 \mathrm{~nm}$, respectively. The resonance wavelengths were extracted from optical emission spectra of fully fabricated HC-VCSELs biased below threshold at $25{ }^{\circ} \mathrm{C}$.

HC-VCSELs were fabricated using a standard process for oxide-confined VCSELs after removal of the GaAs substrate using mechanical thinning for the first part and selective wet etching for the remaining part of the substrate [10]. Ti/Pt/Au

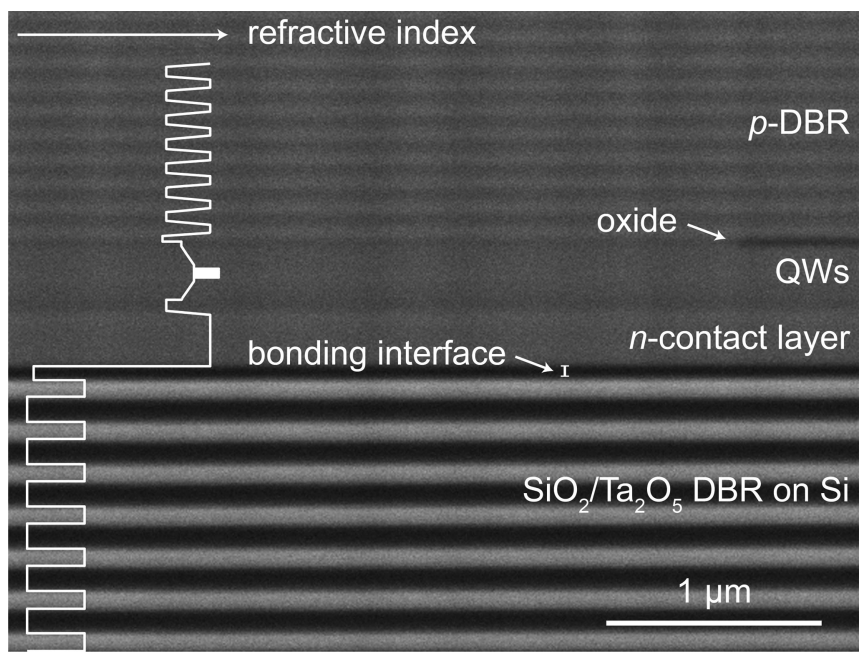

Fig. 5. SEM micrograph of a FIB cross-section through a HC-VCSEL with an overlay of the refractive index profile. The bonding interface between the III-V "half-VCSEL" and $\mathrm{SiO}_{2} / \mathrm{Ta}_{2} \mathrm{O}_{5} \mathrm{DBR}$ on $\mathrm{Si}$ is indicated.

contact rings and alignment marks were evaporated on the top highly $p$-doped GaAs contact layer. The devices were isolated by etching trenches through the III-V using inductively coupled plasma reactive ion etching (ICP-RIE) with $\mathrm{SiCl}_{4}$ chemistry. This was done to allow residual gas trapped at the bonding interface to escape during subsequent high-temperature processing steps. Circular mesas were then etched using the same dry etching technique. An in-situ end-point detection system was used to accurately stop within the thin $(\sim 280 \mathrm{~nm}) \mathrm{Al}_{0.12} \mathrm{Ga}_{0.88} \mathrm{As}$ $n$-contact layer and expose the $\mathrm{Al}_{0.98} \mathrm{Ga}_{0.02}$ As layer for oxidation. The mesa surface and the $n$-contact layer were protected during oxidation by a $100 \mathrm{~nm}$ thick $\mathrm{Si}_{\mathrm{x}} \mathrm{N}_{\mathrm{y}}$ layer deposited by PECVD. Just before oxidation, this layer was removed on the mesa side walls using ICP etching with $\mathrm{NF}_{3}$ chemistry. The oxide formation was performed at $420{ }^{\circ} \mathrm{C}$ in a wet oxidation furnace with in-situ monitoring of the oxidation front. After oxidation, the protective $\mathrm{Si}_{\mathrm{x}} \mathrm{N}_{\mathrm{y}}$ layer was removed using ICP etching with $\mathrm{NF}_{3}$ chemistry to allow evaporation of $\mathrm{Ni} / \mathrm{Ge} / \mathrm{Au}$ intra-cavity $n$-contacts, which were annealed $30 \mathrm{~s}$ at $430{ }^{\circ} \mathrm{C}$. Finally, the mesas were planarized with a thick BCB layer to allow sputtering of low capacitance Ti/Au ground-signal-ground (GSG) bondpads with $100 \mu \mathrm{m}$ pitch.

In Fig. 5, a scanning electron microscope (SEM) micrograph of a focused ion beam (FIB) cross-section through a fully fabricated HC-VCSEL is shown with an overlay of the refractive index profile. The micrograph clearly shows that the III-V "half-VCSEL" is attached to the $\mathrm{SiO}_{2} / \mathrm{Ta}_{2} \mathrm{O}_{5}$ DBR on $\mathrm{Si}$ at the indicated bonding interface.

\section{MEASUREMENTS}

\section{A. Static Performance}

Four HC-VCSELs denoted A-D, with resonance wavelengths of $843,853,861$, and $867 \mathrm{~nm}$ were investigated. The diameter of the oxide aperture is $10 \mu \mathrm{m}$. The output power and voltage versus current measured at ambient temperatures ranging from 

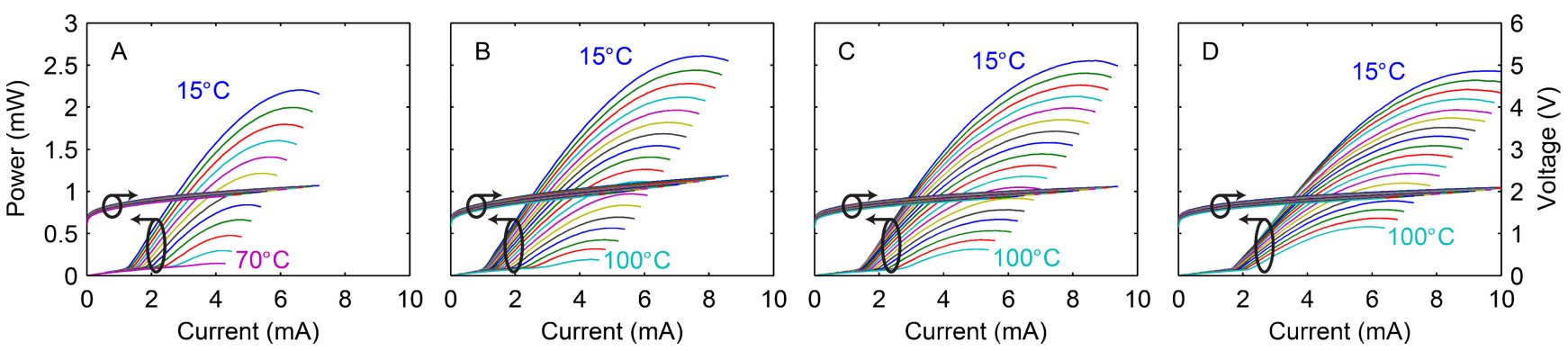

Fig. 6. Output power and voltage versus current for HC-VCSELs A-D measured at ambient temperatures ranging from 15 to $100{ }^{\circ} \mathrm{C}$ in steps of $5{ }^{\circ} \mathrm{C}$.

TABLE II

Static Performance Parameters

\begin{tabular}{|c|c|c|c|c|c|}
\hline \multirow[b]{2}{*}{ Parameter } & \multirow[b]{2}{*}{ Temp. $\left({ }^{\circ} \mathrm{C}\right)$} & \multicolumn{4}{|c|}{ HC-VCSEL } \\
\hline & & A & B & $\mathrm{C}$ & $\mathrm{D}$ \\
\hline Resonance wavelength (nm) & 25 & 843 & 853 & 861 & 867 \\
\hline Peak gain-to-resonance detuning (nm) & 25 & +9 & -1 & -9 & -15 \\
\hline Temperature at min. threshold current $\left({ }^{\circ} \mathrm{C}\right)$ & & 6 & 27 & 36 & 54 \\
\hline \multirow{4}{*}{ Threshold current (mA) } & 25 & 1.42 & 1.07 & 1.42 & 1.85 \\
\hline & 55 & 2.18 & 1.36 & 1.52 & 1.64 \\
\hline & 85 & $\mathrm{n} / \mathrm{a}$ & 2.10 & 2.08 & 1.87 \\
\hline & 100 & $\mathrm{n} / \mathrm{a}$ & 3.00 & 2.77 & 2.24 \\
\hline \multirow[t]{4}{*}{ Maximum optical output power (mW) } & 25 & 1.8 & 2.3 & 2.3 & 2.2 \\
\hline & 55 & 0.66 & 1.4 & 1.4 & 1.5 \\
\hline & 85 & $\mathrm{n} / \mathrm{a}$ & 0.56 & 0.67 & 0.89 \\
\hline & 100 & $\mathrm{n} / \mathrm{a}$ & 0.19 & 0.31 & 0.58 \\
\hline \multirow[t]{4}{*}{ Thermal roll-over current $(\mathrm{mA})$} & 25 & 6.2 & 7.4 & 8.2 & 9.0 \\
\hline & 55 & 4.8 & 6.2 & 7.1 & 7.9 \\
\hline & 85 & $\mathrm{n} / \mathrm{a}$ & 5.0 & 5.7 & 6.6 \\
\hline & 100 & $\mathrm{n} / \mathrm{a}$ & 4.3 & 5.0 & 5.9 \\
\hline \multirow[t]{4}{*}{ Slope efficiency (W/A) } & 25 & 0.54 & 0.55 & 0.54 & 0.50 \\
\hline & 55 & 0.30 & 0.42 & 0.41 & 0.39 \\
\hline & 85 & $\mathrm{n} / \mathrm{a}$ & 0.25 & 0.26 & 0.28 \\
\hline & 100 & $\mathrm{n} / \mathrm{a}$ & 0.09 & 0.16 & 0.22 \\
\hline \multirow[t]{4}{*}{ Differential resistance at $4 \mathrm{~mA}(\Omega)$} & 25 & 61 & 81 & 55 & 51 \\
\hline & 55 & 62 & 79 & 54 & 50 \\
\hline & 85 & $\mathrm{n} / \mathrm{a}$ & 79 & 55 & 50 \\
\hline & 100 & $\mathrm{n} / \mathrm{a}$ & 81 & 55 & 51 \\
\hline \multirow[t]{4}{*}{ Differential resistance at thermal roll-over $(\Omega)$} & 25 & 48 & 61 & 33 & 28 \\
\hline & 55 & 60 & 67 & 41 & 34 \\
\hline & 85 & $\mathrm{n} / \mathrm{a}$ & 76 & 48 & 42 \\
\hline & 100 & $\mathrm{n} / \mathrm{a}$ & 81 & 53 & 45 \\
\hline
\end{tabular}

15 to $100^{\circ} \mathrm{C}$, in steps of $5{ }^{\circ} \mathrm{C}$, are shown in Fig. 6 while the corresponding static performance characteristics are summarized in Table II.

The variation of threshold current with the bonding interface thickness at $25{ }^{\circ} \mathrm{C}$ follows the variation of threshold gain predicted by the simulations (Fig. 3). Minimum threshold current $(1.1 \mathrm{~mA})$ is obtained for HC-VCSEL B which has the smallest gain-to-resonance detuning at $25{ }^{\circ} \mathrm{C}$. At higher ambient temperatures, HC-VCSELs C and D have lower threshold currents since the detuning is reduced with temperature as a result of the $\sim 4$ times faster redshift of the gain peak with respect to the cavity resonance [15]. The threshold currents from Fig. 6 are shown versus ambient temperature in Fig. 7(a). The temperatures for minimum threshold current were identified by fitting a second order polynomial to the dependence of threshold current on temperature, as seen in Fig. 7(b). At the temperature

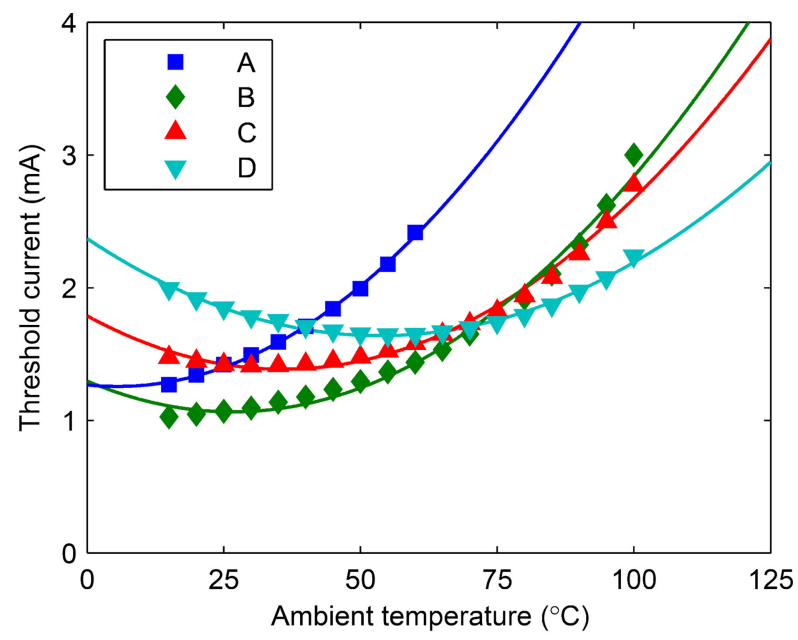

(a)

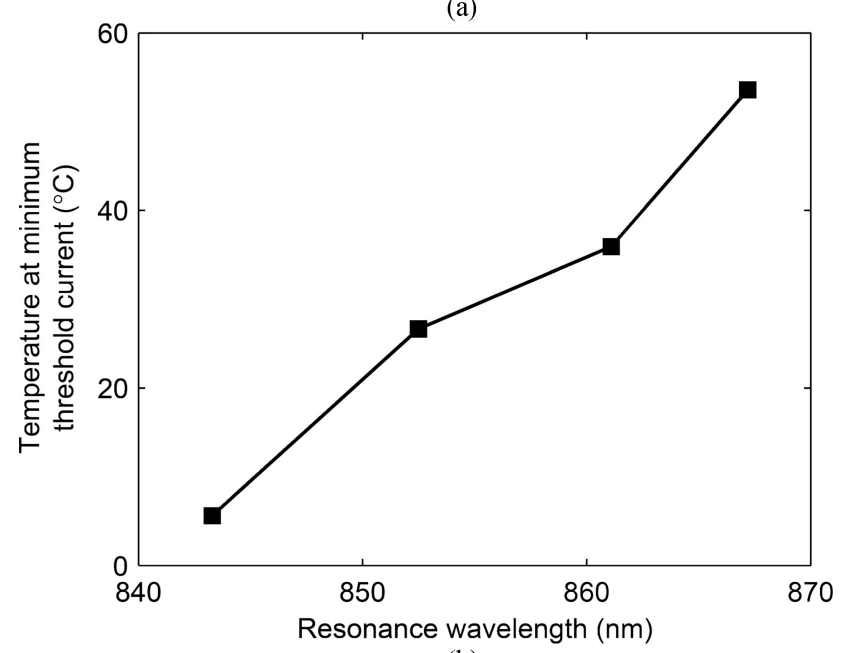

(b)

Fig. 7. (a) Threshold current versus ambient temperature and (b) estimated temperature at minimum threshold current versus resonance wavelength.

for minimum threshold current, the wavelengths of the cavity resonance and the gain peak are aligned. At $25^{\circ} \mathrm{C}$ this occurs at $852 \mathrm{~nm}$ as estimated in Section II. While the threshold current of HC-VCSEL D is higher than that of HC-VCSEL B at $25^{\circ} \mathrm{C}$, it is lower at high temperatures and shows a weaker dependence on temperature. Therefore, the thickness of the bonding interface can be chosen for either minimum threshold current at a specific ambient temperature or a small variation of threshold current over the temperature range. 


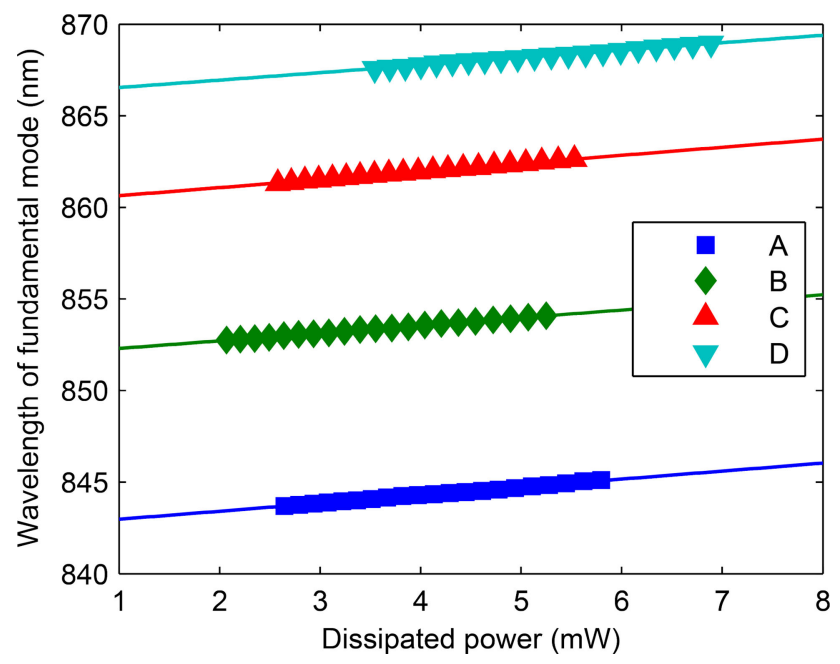

Fig. 8. Wavelength of the fundamental mode versus dissipated power at $25^{\circ} \mathrm{C}$ for HC-VCSELs A-D with fits to extract the thermal impedance.

The achievable output power depends on the threshold current, the slope efficiency and the thermal roll-over current. All HC-VCSELs have similar slope efficiency $(\sim 0.5 \mathrm{~W} / \mathrm{A}$ at $25^{\circ} \mathrm{C}$ ), with a slight variation caused by an uncertainty in the thickness of the topmost $p$-DBR layer after substrate removal. At high temperatures, the slope efficiency is reduced as a result of increased internal optical loss (free carrier absorption) and reduced internal quantum efficiency [16]. The thermal roll-over current is largely determined by the thermal impedance and the dependence of threshold current on temperature since the internal temperature increases with current due to heat dissipation caused by the electrical resistance and the free carrier absorption [16]. Therefore, while HC-VCSEL B and C produce the highest output power at $25{ }^{\circ} \mathrm{C}(2.3 \mathrm{~mW}), \mathrm{HC}-\mathrm{VCSEL} \mathrm{D}$ shows improved high temperature and high current performance since the gain peak aligns with the resonance wavelength at higher internal temperature. The maximum output power at $100{ }^{\circ} \mathrm{C}$ is $0.6 \mathrm{~mW}$. The rapid increase of threshold current with temperature for HC-VCSEL A limits the maximum operating temperature to $70{ }^{\circ} \mathrm{C}$. Again, the thickness of the bonding interface can be chosen for either optimum performance at a specific ambient temperature or a small variation in performance over the temperature range.

Thermal impedance was measured by tracking the wavelength shift of the fundamental mode with dissipated power and ambient temperature [17]. The fundamental mode redshifts with temperature by $0.059 \mathrm{~nm} / \mathrm{K}$, while it redshifts with dissipated power by $0.42 \mathrm{~nm} / \mathrm{mW}$ (Fig. 8). This gives a thermal impedance of $7 \mathrm{~K} / \mathrm{mW}$ for all HC-VCSELs, regardless of the thickness of the bonding interface. This indicates that the thermal impedance is to a large extent determined by heat transport through the dielectric DBR. With the thermal impedance being $\sim 4$ times larger than for ordinary GaAs-based oxide-confined VCSELs [16], the thermal roll-over currents are relatively low, which limits the maximum output power.

The differential resistances are comparable (50-60 $\Omega$ ), with negligible dependence on temperature, since the bonding

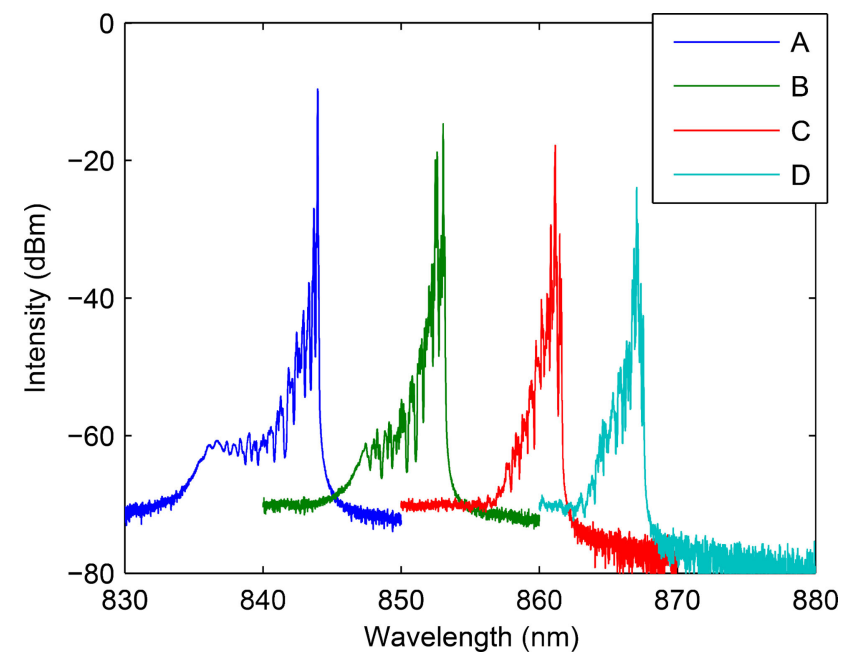

Fig. 9. Emission spectra at $25^{\circ} \mathrm{C}$ for HC-VCSELs A-D biased at $2 \mathrm{~mA}$.

interface thickness should have no impact on the electrical characteristics. The somewhat higher resistance of HC-VCSEL B $(80 \Omega)$ is believed to be due to residues from the removal of the substrate, which led to a higher $p$-contact resistance. The performance of HC-VCSEL B is still superior in terms of maximum output power at temperatures up to $25^{\circ} \mathrm{C}$.

Optical emission spectra, measured at a bias current of $2 \mathrm{~mA}$, are shown in Fig. 9. The dependence of wavelength on the bonding interface thickness agrees well with the simulations in Fig. 3. Also the modal characteristics are affected by the bonding interface thickness, which controls the gain-to-resonance detuning, with the lower order (longer wavelength) modes favored by positive detuning and higher order (shorter wavelength) modes by negative detuning.

\section{B. Dynamic Performance}

The dependence of the dynamic performance on bonding interface thickness/detuning was investigated by recording the small-signal modulation response at 25,55 , and $85^{\circ} \mathrm{C}$. Measurements were performed using a $67 \mathrm{GHz}$ vector network analyzer (Rohde \& Schwarz ZVA67), with internal bias-T, connected to the HC-VCSEL with a high-speed probe (Picoprobe 40AGSG-100P) matching the HC-VCSEL bondpad pitch. The output was coupled to a $1 \mathrm{~m}$ long OM4 multimode optical fiber through an antireflection (AR) coated lens package, followed by a $25 \mathrm{GHz}$ photodetector (New Focus 1481-S-50) and the network analyzer. A variable optical attenuator (EXFO FVA-3150) was inserted before the photodetector to avoid saturation of the photodetector.

The modulation response for HC-VCSELs A-D at 25 and $85^{\circ} \mathrm{C}$, compensated for the frequency response of the probe and the photodetector, are shown in Fig. 10. A three-pole transfer function [18] was fitted to the measured modulation response to extract the resonance frequency and damping rate, as well as the parasitic pole frequency. The dynamic performance characteristics of all HC-VCSELs at three ambient temperatures are summarized in Table III. 


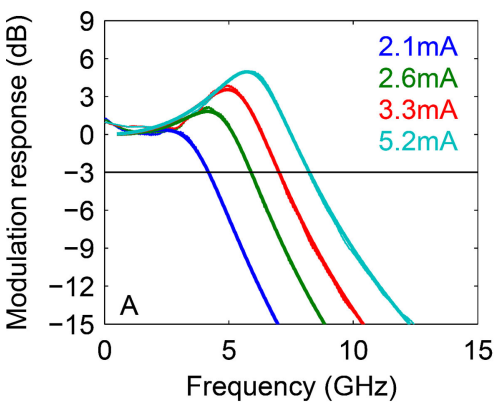

(a)

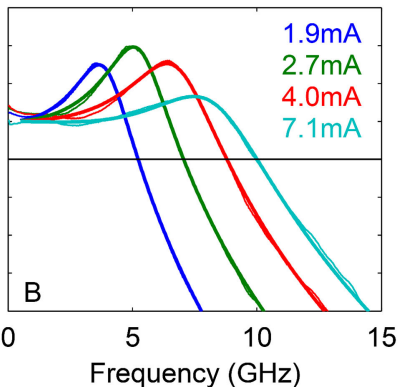

(b)

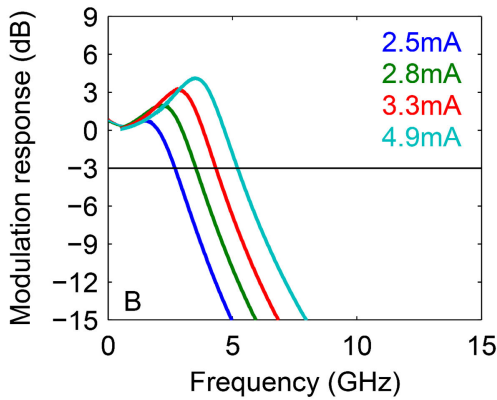

(e)

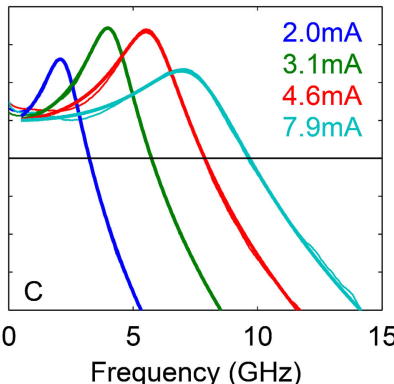

(c)

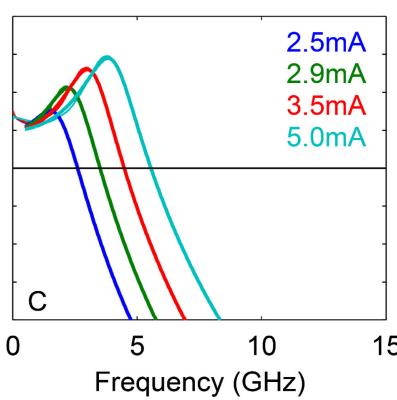

(f)

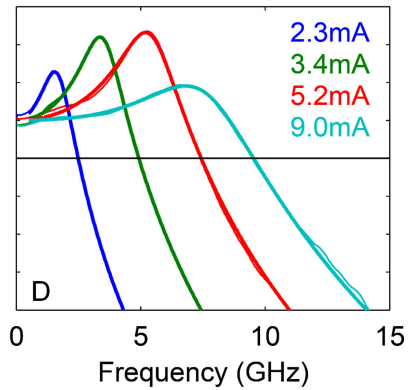

(d)

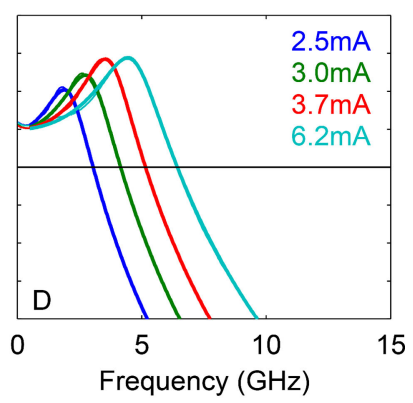

(g)

Fig. 10. Small-signal modulation response at $25{ }^{\circ} \mathrm{C}$ (a)-(d) and $85^{\circ} \mathrm{C}(\mathrm{e})-(\mathrm{g})$ for HC-VCSELs A-D at indicated bias currents. The maximum $3 \mathrm{~dB}$ modulation bandwidth is reached at the highest bias currents indicated.

TABLE III

DYNAMIC PERFORMANCE PARAMETERS

\begin{tabular}{|c|c|c|c|c|c|}
\hline \multirow[b]{2}{*}{ Parameter } & \multirow[b]{2}{*}{ Temp. $\left({ }^{\circ} \mathrm{C}\right)$} & \multicolumn{4}{|c|}{ HC-VCSEL } \\
\hline & & A & $\mathrm{B}$ & $\mathrm{C}$ & $\mathrm{D}$ \\
\hline \multirow[t]{3}{*}{ Maximum $3 \mathrm{~dB}$ bandwidth $(\mathrm{GHz})$} & 25 & 8.2 & 10.0 & 9.7 & 9.5 \\
\hline & 55 & 4.3 & 8.1 & 8.1 & 8.1 \\
\hline & 85 & $\mathrm{n} / \mathrm{a}$ & 5.2 & 5.6 & 6.4 \\
\hline \multirow[t]{3}{*}{ Bias current at max. $3 \mathrm{~dB}$ bandwidth (mA) } & 25 & 5.2 & 7.1 & 7.9 & 9.0 \\
\hline & 55 & 4.4 & 5.8 & 6.9 & 7.1 \\
\hline & 85 & $\mathrm{n} / \mathrm{a}$ & 4.9 & 5.0 & 6.2 \\
\hline \multirow[t]{3}{*}{$D$-factor $\left(\mathrm{GHz} / \mathrm{mA}^{1 / 2}\right)$} & 25 & 4.8 & 4.2 & 3.3 & 3.0 \\
\hline & 55 & 2.8 & 4.2 & 3.3 & 2.9 \\
\hline & 85 & $\mathrm{n} / \mathrm{a}$ & 3.1 & 2.6 & 2.7 \\
\hline
\end{tabular}

All HC-VCSELs show a strongly resonant modulation response caused by the relatively low photon density established in the cavity at thermal roll-over. A more damped response is desired to reduce overshoot and jitter during large signal modulation and data transmission [19].

Although the modulation bandwidth is largely limited by the capacitance over the single oxide layer (with a parasitic pole frequency of 6-8 GHz), there are clear differences in the maximum modulation bandwidth related to the differences in bonding interface thickness and gain-to-resonance detuning. The maximum modulation bandwidth depends on how fast the resonance frequency increases with bias current (quantified by the $D$-factor) and the highest photon density that can be achieved [20].

The $D$-factors were extracted from the measured dependence of the resonance frequency on the square root of current above threshold, as shown in Fig. 11(a) at $25^{\circ} \mathrm{C}$. The $D$-factors are plotted in Fig. 11(b). Clearly, the $D$-factor is reduced with increasing resonance wavelength. This is caused by the differential gain being lower on the long-wavelength side of the gain peak. At higher temperatures (Table III), the $D$-factors are further reduced due to a reduction of differential gain with temperature and a more pronounced reduction of internal quantum efficiency at the highest temperature.

The maximum photon density is reached at the thermal rollover current. Therefore, higher photon densities can be established in HC-VCSELs with low threshold current and weak dependence of threshold current on temperature.

As a consequence of these dependencies, HC-VCSEL B has the highest $3 \mathrm{~dB}$ modulation bandwidth at $25{ }^{\circ} \mathrm{C}(10.0$ $\mathrm{GHz}$ ) while HC-VCSEL D has the highest bandwidth at $85{ }^{\circ} \mathrm{C}$ (6.4 GHz). HC-VCSEL D also shows the smallest dependence of bandwidth on temperature. Similar to the threshold current and the maximum output power, the bonding interface thickness can be chosen for either a maximum bandwidth at a specific ambient temperature or a more temperature-stable bandwidth.

\section{Data Transmission}

A HC-VCSEL with similar resonance wavelength $(859 \mathrm{~nm})$ as HC-VCSEL C, but with a smaller oxide aperture diameter $(5 \mu \mathrm{m})$, was chosen for back-to-back data transmission experiments. The smaller aperture device has a slightly more damped modulation response and a higher photon density at low bias currents. The small signal $3 \mathrm{~dB}$ modulation bandwidth for this device is 12.1 and $8.9 \mathrm{GHz}$ at 25 and $85^{\circ} \mathrm{C}$, respectively.

A non-return-to-zero pseudo random bit sequence (PRBS) signal with word length $2^{7}-1$ was generated by a bit pattern 


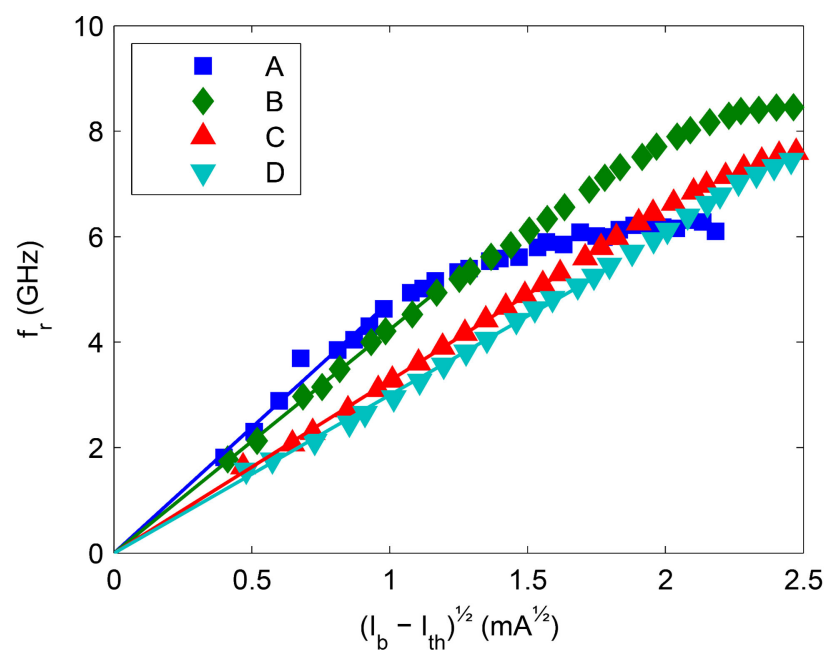

(a)

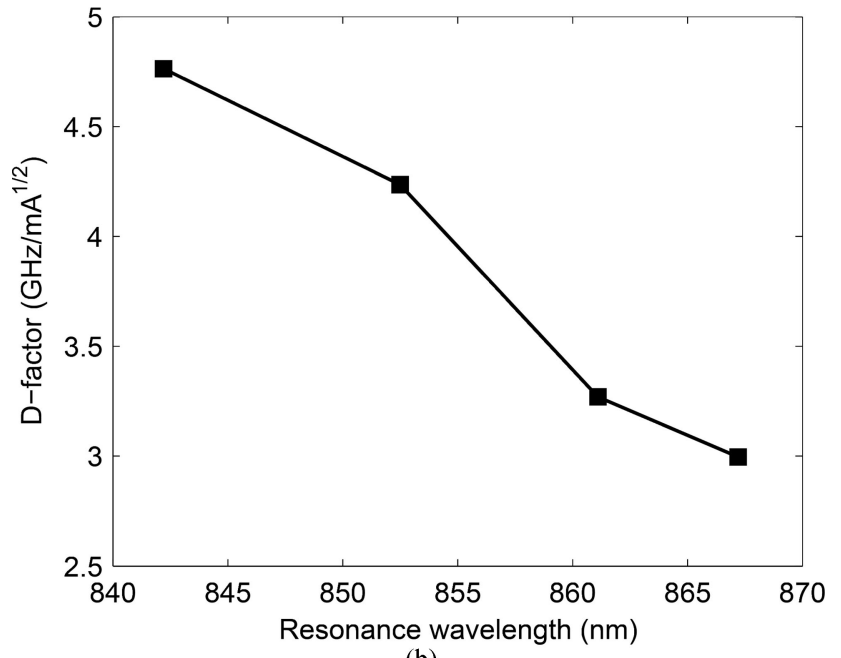

(b)

Fig. 11. (a) Resonance frequency versus square root of current above threshold at $25^{\circ} \mathrm{C}$ with fits to extract the $D$-factors, which are shown versus resonance wavelength in (b).

generator (SHF 12103A) and fed to the HC-VCSEL through the high-speed probe. A high-speed bias-T was inserted before the probe to allow DC biasing of the HC-VCSEL. The light from the HC-VCSEL was coupled into a $1 \mathrm{~m}$ long OM4 multimode optical fiber using the AR coated lens package. The fiber was connected to a $30 \mathrm{GHz}$ limiting photoreceiver (VI Systems R40850) via a variable optical attenuator. The photoreceiver was finally connected to either an error analyzer (SHF 11100B) to measure the bit error ratio (BER) or a $70 \mathrm{GHz}$ equivalent time sampling oscilloscope (Agilent Infiniium DCA-J 86100C) to record eye diagrams.

With a bias current of $4.5 \mathrm{~mA}$, a modulation voltage of $350 \mathrm{mV}_{\mathrm{pp}}$, and the HC-VCSEL held at $25^{\circ} \mathrm{C}$, error-free transmission (BER $<10^{-12}$ ) was achieved at data rates up to $25 \mathrm{~Gb} / \mathrm{s}$ with an extinction ratio (ER) of $5.6 \mathrm{~dB}$ (Fig. 12). At $85^{\circ} \mathrm{C}$, a bias current of $3.5 \mathrm{~mA}$ and modulation voltage of $180 \mathrm{mV}_{\mathrm{pp}}$ enabled error-free transmission at data rates up to $10 \mathrm{~Gb} / \mathrm{s}(\mathrm{ER}=5.9 \mathrm{~dB})$. The lower bias current and modulation voltage used at $85^{\circ} \mathrm{C}$ were required to maintain high modulation

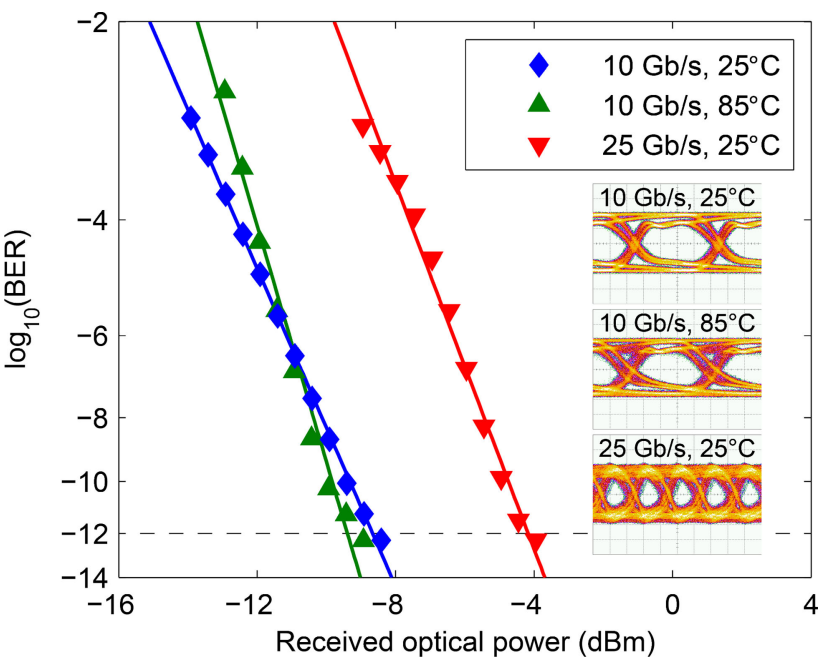

Fig. 12. Measured BER versus received optical power for a 5- $\mu$ m oxideaperture diameter $\mathrm{HC}$-VCSEL with similar resonance wavelength as $\mathrm{HC}$ VCSEL $\mathrm{C}$ at data rates up to $25 \mathrm{~Gb} / \mathrm{s}$ at $25^{\circ} \mathrm{C}$ and $10 \mathrm{~Gb} / \mathrm{s}$ at $85^{\circ} \mathrm{C}$. Insets: Corresponding optical eye diagrams (scales: $100 \mathrm{mV} / \mathrm{div}$ and $20 \mathrm{ps} / \mathrm{div}$ ).

efficiency and avoid operation below threshold at the off-state. The same biasing and modulation conditions were also used with the HC-VCSEL held at $25{ }^{\circ} \mathrm{C}$ and modulated at $10 \mathrm{~Gb} / \mathrm{s}$ for comparison, resulting in a reduction of the ER to $3.9 \mathrm{~dB}$ due to the lower threshold current. At $10 \mathrm{~Gb} / \mathrm{s}$, there is no power penalty when increasing the temperature from 25 to $85^{\circ} \mathrm{C}$ due to the higher ER at $85^{\circ} \mathrm{C}$, but the power budget is $2.3 \mathrm{~dB}$ larger at $25^{\circ} \mathrm{C}$. However, increasing the data rate from 10 to $25 \mathrm{~Gb} / \mathrm{s}$ at $25^{\circ} \mathrm{C}$ results in a $4 \mathrm{~dB}$ power penalty.

\section{CONCLUSION}

By varying the bonding interface thickness of siliconintegrated hybrid-cavity VCSELs we have studied its impact on important static and dynamic performance parameters. The variable thickness offers the opportunity to either optimize performance (e.g. threshold current, output power, modulation bandwidth) at a specific ambient temperature or optimize for more temperature-stable performance.

With a $10-\mu \mathrm{m}$ oxide-aperture diameter we reach an output power (modulation bandwidth) of $2.3 \mathrm{~mW}(10.0 \mathrm{GHz})$ at $25^{\circ} \mathrm{C}$ and $0.9 \mathrm{~mW}(6.4 \mathrm{GHz})$ at $85^{\circ} \mathrm{C}$ for HC-VCSELs with different interface thicknesses and detuning. The HC-VCSEL with the largest detuning of $-15 \mathrm{~nm}$ (thickest bonding interface) also shows weak temperature dependencies of the threshold current and the modulation bandwidth over the temperature range $25-85^{\circ} \mathrm{C}$. We also found the thermal impedance to be independent of the thickness of the bonding interface, therefore being largely determined by the thermal conductivity of the dielectric DBR. Finally, we demonstrated error-free data transmission back-to-back at bit rates up to 25 and $10 \mathrm{~Gb} / \mathrm{s}$ at 25 and $85^{\circ} \mathrm{C}$, respectively, using a 5- $\mu$ m oxide-aperture diameter HC-VCSEL with moderately large gain-to-resonance detuning. 


\section{REFERENCES}

[1] Z. Zhou, B. Yin, and J. Michel, "On-chip light sources for silicon photonics," Light Sci. Appl., vol. 4, no. 11, Nov. 2015, Paper no. e358.

[2] K. Takaki et al., "A recorded 62\% PCE and low series and thermal resistance VCSEL with a double intra-cavity structure," in Proc. IEEE Int. Semicond. Laser Conf., 2008, Paper no. PDP1.

[3] Y. Wang et al., "Vertical-cavity surface-emitting laser flip-chip bonding to silicon photonics chip," in Proc. IEEE Opt. Interconnects Conf., 2015, vol. 6, pp. 122-123.

[4] R. Chen et al., "Nanolasers grown on silicon," Nature Photon., vol. 5 , no. 3, pp. 170-175, Mar. 2011.

[5] S. Keyvaninia et al., "Ultra-thin DVS-BCB adhesive bonding of III-V wafers, dies and multiple dies to a patterned silicon-on-insulator substrate," Opt. Mater. Express, vol. 3, no. 1, pp. 35-46, Jan. 2013.

[6] Y. Tsunemi et al., "1.55- $\mu \mathrm{m}$ VCSEL with polarization-independent HCG mirror on SOI," Opt. Express, vol. 21, no. 23, pp. 28685-28692, Nov. 2013

[7] J. Ferrara, W. Yang, L. Zhu, P. Qiao, and C. J. Chang-Hasnain, "Heterogeneously integrated long-wavelength VCSEL using silicon high contrast grating on an SOI substrate," Opt. Express, vol. 23, no. 3, pp. 2512-2523, Feb. 2015.

[8] G. C. Park et al., "Hybrid vertical-cavity laser with lateral emission into a silicon waveguide," Laser Photon. Rev., vol. 9, no. 3, pp. L11-L15, May 2015.

[9] D. A. Louderback et al., "VCSELs with monolithic coupling to internal horizontal waveguides using integrated diffraction gratings," Electron. Lett., vol. 40, no. 17, pp. 1064-1065, Aug. 2004

[10] E. P. Haglund et al., "Silicon-integrated short-wavelength hybrid-cavity VCSEL," Opt. Express, vol. 23, no. 26, pp. 33634-33640, Dec. 2015.

[11] E. P. Haglund et al., "20-Gb/s modulation of silicon-integrated shortwavelength hybrid-cavity VCSELs," IEEE Photon. Technol. Lett., vol. 28, no. 8, pp. 856-859, Apr. 2016.

[12] D. B. Young et al., "Enhanced performance of offset-gain high-barrier vertical-cavity surface-emitting lasers," IEEE J. Quantum Electron., vol. 29, no. 6, pp. 2013-2022, Jun. 1993.

[13] H. Li et al., "Impact of the quantum well gain-to-cavity etalon wavelength offset on the high temperature performance of high bit rate $980-\mathrm{nm}$ VCSELs," IEEE J. Quantum Electron., vol. 50, no. 8, pp. 613-621, Aug. 2014.

[14] G. R. Hadley, "Effective index model for vertical-cavity surface-emitting lasers," Opt. Lett., vol. 20, no. 13, pp. 1483-1485, Jul. 1995.

[15] R. Michalzik, "VCSEL fundamentals," in VCSELs: Fundamentals, Technology and Applications of Vertical-Cavity Surface-Emitting Lasers, R. Michalzik, Ed. Berlin, Heidelberg: Springer, 2013, pp. 19-75.

[16] P. P. Baveja et al., "Assessment of VCSEL thermal rollover mechanisms from measurements and empirical modeling," Opt. Express, vol. 19, no. 16, pp. 15490-15505, Aug. 2011.

[17] W. Nakwaski, "Thermal aspects of efficient operation of vertical-cavity surface-emitting lasers," Opt. Quantum Electron., vol. 28, no. 4, pp. 335352, Apr. 1996.

[18] L. Coldren and S. Corzine, Diode Lasers and Photonic Integrated Circuits. New York, NY, USA: Wiley, 1995.

[19] E. P. Haglund, P. Westbergh, J. S. Gustavsson, and A. Larsson, "Impact of damping on high-speed large signal VCSEL dynamics," J. Light. Technol., vol. 33, no. 4, pp. 795-801, Feb. 2015.

[20] A. Larsson, "Advances in VCSELs for communication and sensing," IEEE J. Sel. Topics Quantum Electron., vol. 17, no. 6, pp. 1552-1567, Nov. 2011.

Emanuel P. Haglund (S'14) received the M.Sc. degree in engineering physics in 2013 from Chalmers University of Technology, Göteborg, Sweden, where he is currently working toward the Ph.D. degree in microtechnology and nanoscience at the Photonics Laboratory.

His current research interests include fabrication and characterization of silicon-integrated GaAs-based hybrid vertical-cavity lasers and high-speed vertical-cavity surface-emitting lasers for future optical interconnects in data centers and high-performance computers.
Sulakshna Kumari received the five year Integrated M.Sc. degree in photonics from Cochin University of Science and Technology, Kerala, India, in 2012. Since 2012, she has been working toward the Ph.D. degree in the Department of Information Technology (INTEC), Ghent University, Gent, Belgium.

She did her Master's thesis work at the National University of Singapore, Singapore, in 2012

Her current research interests include integration of GaAs vertical-cavity surface-emitting lasers on top of silicon nitride waveguide circuits.

Erik Haglund (S'12-M'15) received the M.Sc. degree in engineering physics in 2010 and the Ph.D. degree in microtechnology and nanoscience in 2015, both from Chalmers University of Technology, Göteborg, Sweden.

His thesis focused on the design, fabrication, and characterization of highspeed vertical-cavity surface-emitting lasers (VCSELs) for fiber-optical links (optical interconnects) in future data centers and high-performance computing. He is currently working with short-wavelength VCSELs for optical communication and sensors in the Department of Microtechnology and Nanoscience, Chalmers University of Technology. His research interests include higher bandwidth VCSELs, energy-efficient data transmission, and spectral engineering of VCSELs using surface structures such as high-contrast gratings.

Johan S. Gustavsson received the M.Sc. degree in electrical engineering and the Ph.D. degree in photonics from the Chalmers University of Technology, Göteborg, Sweden, in 1998 and 2003, respectively. His main research topics were mode dynamics and noise in vertical-cavity surface-emitting lasers (VCSELs).

Since 2003, he has been a Researcher in the Photonics Laboratory, Department of Microtechnology and Nanoscience, Chalmers University of Technology, with an Assistant Professor position (2004-2008), and an Associate Professor position since 2011. From September to October 2009, he was a Visiting Scientist at CNR Polytechnico, Turin, Italy. He has authored or coauthored more than 180 scientific journal and conference papers, and his research has been focused on semiconductor lasers for short to medium reach communication, and sensing applications. This has included surface relief techniques for mode and polarization control in VCSELs, $1.3 \mu \mathrm{m}$ InGaAs VCSELs, GaInNAs ridge waveguide lasers for access networks, 2.3-3.5 $\mu \mathrm{m} \mathrm{GaSb}$ VCSELs for $\mathrm{CO}, \mathrm{CO}_{2}$, and $\mathrm{NH}_{3}$ sensing, and tunable VCSELs via moveable mirror for reconfigurable optical interconnects. He is currently working on energy efficient $56 \mathrm{~Gb} / \mathrm{s} \mathrm{GaAs}$ based VCSELs for next-generation datacom links, blue/green GaN VCSELs, high contrast gratings as feedback and wavelength setting elements in microcavity lasers, and heterogeneous integration of III/V-based VCSEL material on $\mathrm{Si}$ platform.

Roel G. Baets (M'88-SM'96-F'07) received the M.Sc. degree in electrical engineering from Ghent University (UGent), Ghent, Belgium, the M.Sc. degree from Stanford University, Stanford, CA, and the Ph.D. degree from UGent in 1980, 1981, and 1984, respectively.

From 1984 to 1989, he held a postdoctoral position at IMEC (with detachment to Ghent University). From 1990 to 1994, he was a Part-Time Professor at the Technical University of Delft, and from 2004 to 2008, at the Technical University of Eindhoven. Since 1989, he has been a Professor in the Engineering Faculty of UGent, where he founded the Photonics Research Group. He is currently a Full Professor at UGent. He is also associated with IMEC. He has management responsibilities within the Photonics Research Group, UGent, the Center for Nano- and Biophotonics, UGent, and the joint UGent-IMEC research program on silicon photonics.

He has mainly worked in the field of integrated photonic components and circuits. He has made contributions to research on semiconductor laser diodes, guided wave and grating devices and to the design and fabrication of photonic ICs, both in III-V semiconductors and in silicon. In recent years, his research has focused on spectroscopic sensing applications of silicon photonics (both SOI and silicon nitride).

Prof. Baets is the coauthor of more than 700 ISI-WoS listed journal and conference papers. He is a grant holder of the Methusalem Programme of the Flemish government and of the European Research Council (ERC advanced grant). 
Gunther Roelkens (M'07) received a degree in electrical engineering from Ghent University, Ghent, Belgium, in 2002, and the Ph.D. degree in 2007 from the Department of Information Technology, Ghent University, where he is currently an Associate Professor. In 2008, he was a Visiting Scientist in the IBM TJ Watson Research Center, New York, NY, USA. He is currently an Assistant Professor at Eindhoven University of Technology, Eindhoven, The Netherlands. His research interests include the heterogeneous integration of III-V semiconductors and other materials on top of silicon waveguide circuits and electronic/photonic cointegration. He received an ERC starting grant (MIRACLE), to start up research in the field of integrated midinfrared photonic integrated circuits. He is the coauthor of more than 150 papers in peer-reviewed journals and 300 conference papers.
Anders Larsson (M'88-SM'09-F'14) received the M.Sc. and Ph.D. degrees in electrical engineering from Chalmers University of Technology, Göteborg, Sweden, in 1982 and 1987, respectively.

In 1991, he joined the faculty at Chalmers where he became a Professor in 1994. From 1984 to 1985, he was with the Department of Applied Physics, California Institute of Technology, Pasadena, CA, USA, and from 1988 to 1991 with the Jet Propulsion Laboratory, Pasadena, CA, USA. He has been a Guest Professor at Ulm University, Ulm, Germany, in the Optical Science Center, University of Arizona, Tucson, AZ, USA, at Osaka University, Suita, Japan, and in the Institute of Semiconductors, Chinese Academy of Sciences, Beijing, China. His scientific background is in the areas of optoelectronic materials and devices for optical communication, information processing, and sensing. His current research is focused on vertical-cavity surface-emitting lasers and optical interconnects. He has published more than 500 scientific journal and conference papers and 2 book chapters. He co-organized the IEEE Semiconductor Laser Workshop 2004 and organized the European Semiconductor Laser Workshop 2004.

Prof. Larsson was a Co-Program Chair for the European Conference on Optical Communication 2004, and was the Program and General chair for the IEEE International Semiconductor Laser Conference in 2006 and 2008, respectively. He is a member of the IEEE Photonics Society Board of Governors, an Associate Editor for IEEE/OSA JOURNAL OF LIGHTWAVE TECHNOLOGY, and a member of the editorial board of IET Optoelectronics. He is a Fellow of OSA and EOS. In 2012, he received the HP Labs Research Innovation Award. 